\title{
Maintenance of continuity of care in a rotating-physician model in an outpatient palliative care clinic
}

\author{
Debbie Selby ${ }^{*}$, Audrey Kim², Anita Chakraborty ${ }^{1}$ \\ ${ }^{1}$ Palliative Care Consult Team, Sunnybrook Health Sciences Centre \\ ${ }^{2}$ University of Toronto, Ontario Canada
}

Received: August 30, 2015; Accepted: October 27, 2015; Published: November 09, 2015

*Corresponding author: Debbie Selby, Palliative Care Consult Team, Sunnybrook Health Sciences Centre, Rm H336, 2075 Bayview Avenue, Toronto, Ontario, Canada, phone :416 4806100 x7255, fax :416 480 5146; E-mail : Debbie.Selby@sunnybrook.ca

\begin{abstract}
Palliative care is a field in which continuity of care (COC) may be seen as particularly relevant, given its role in providing comprehensive and compassionate full person care for patients with advanced illnesses. The role of continuity of physician care in this setting has not been fully explored. The purpose of this study was to explore patient concerns both before and after a scheduled rotation of palliative care physicians in an outpatient clinic, with a particular focus on the patients' perception of impact on their care.

Recruited patients from clinic $(\mathrm{N}=18)$ participated in qualitative interviews following the change in provider in clinic. Six key topics were explored with themes identified for each : 1) How the change was communicated; 2) Reaction to hearing about the change; 3) Feelings after meeting the new physician; 4) Factors felt to help transition; 5) Overall impact on care; 6) Suggestions for future transitions.
\end{abstract}

\section{Introduction}

Palliative care aims to provide comprehensive and compassionate care focusing on symptom management and end of life care for patients with advanced illnesses. While the structure and utility of inpatient palliative care services, hospices, and palliative care units have been well studied $*[1,2]$, more recent attention has focused on the delivery of ambulatory palliative care services $[3,4]$.

Continuity of care (COC) has been defined as "the degree to which a series of discrete healthcare events is experienced as coherent and connected and consistent with a patient's medical needs and personal context." [5]. COC for patients with serious illnesses has long been regarded as important, with attention paid to many facets of continuity, including both personal or relational continuity as well as informational/knowledge continuity [6, 7]. Studies from family practice as well as home-based palliative care indicate the importance of COC for patients $[8,9,10,11]$. A Cochrane review addressed continuity concerns in patients with cancer [12] and commented on the need for further research on not only improving continuity, but also on examining which aspects of the construct are most meaningful to patients.

Palliative care is a field in which continuity may be seen as particularly relevant, given its role in providing comprehensive and compassionate full person care for patients with advanced illnesses. Several studies have documented the benefits of a palliative care service for inpatients in acute care settings $[13,14]$ and outpatients in both clinic and hospice settings $[4,15,16]$. However, the role of continuity of physician care in these settings requires further exploration. A recent study examining lung cancer patients' experiences suggests that better COC is associated with patients having their supportive care needs met, both in the domains of information and psychological support [17]. There is some evidence at the family practice level that continuity of care is important to patients and that changes in physician care can be problematic $[18,19,20]$ however studies specifically examining patient perspectives on physician changes in provision of outpatient palliative care are lacking.

The purpose of this study was to explore patient concerns both before and after a scheduled rotation of palliative care physicians in an outpatient clinic in a large tertiary care cancer center, with a particular focus on the perceived patient impact as well as on factors felt to mitigate any negative impact.

\section{Methods}

Patients were recruited from the Palliative Care Clinic (PCC) at Sunnybrook Health Sciences Center (SHSC) in Toronto, Canada, which is a tertiary care referral center for cancer patients. The PCC was structured in such a way that physicians rotated from the inpatient consultation team to the outpatient clinic in 6 month intervals. A standardized template was developed for the transition, which included:

1. Informing patients of the upcoming change, ideally 1-2 months in advance and in person during a regular appointment.

2. Dictation of a comprehensive summary note on the final visit prior to the rotation. 
3. Direct person-to-person handover to the incoming physician at the discretion of the primary physician (eg patients with particularly complex medical or psychosocial issues).

4. An opportunity to meet the incoming physician where the primary physician felt a personal introduction would ease anxiety.

5. The expectation that incoming physicians would have comprehensively reviewed notes prior to the first visit postrotation.

Other clinic staff (nurses and social worker) remained constant through the physician rotation in an attempt to minimize disruption.

conclusion criteria for patient recruitment included age over 18, fluency in English, metastatic cancer, and attendance at least 2 visits with each physician (at least 2 clinic visits prior to transition and at least 2 with the new physician post transition). All patients provided informed consent with a research assistant not involved with the care process and the study was approved by the Research Ethics Board.

Consenting patients participated in an interview that explored issues around the transition process from the patient perspective. A research assistant not involved with the care process conducted the interviews, and responses were kept confidential from the physicians.

\section{Data analysis}

Themes from the interviews were determined by two independent reviewers (AC, DS) and interviews were coded using NVIVO software. Data saturation was reached when no new themes were emerging.

\section{Results}

Twenty-two eligible patients were approached with 18 agreeing to participate. The median age of recruited patients was 65 (range 49-83) and 12 were female

Six key topics were identified from the interviews and analyzed for themes.

Topic 1: How the change was communicated

Theme 1: Importance of person-to-person communication

Several patients commented on the importance of hearing about the change in person during a clinic visit. One person was notified by phone and found this very upsetting whereas those told during a clinic visit noted that this allowed them the chance to ask questions and generally allayed anxiety. The consistent emerging theme was that the absolute timing of the communication about a change was less important than learning through direct contact with the 'known' physician.

Topic 2: Reaction to hearing about the change

Theme 2: Worries about 'starting over'

Several patients expressed that on hearing of the planned change in provider they were worried about having to 'start all over' with their medical history and that a new physician would not know the key components of their health issues.

Theme 3: Worry about liking the new physician

A few patients stated they were worried about whether or not they would like the new physician.

Theme 4: Importance of understanding why the change was occurring

The most common comment was the importance of having an understanding as to why the change was occurring. Those who had a good sense of this expressed a lower degree of anxiety.

Topic 3: Feelings after meeting the new physician

Theme 5: Importance of a sense of warmth/friendliness

Overall reactions after meeting with the new physician were very positive and recurring comments included the importance of a warm, friendly demeanor of the new physician, which alleviated any anxiety. Many also noted that while their 'old' and 'new' physicians had different styles, these differences were generally welcomed.

Theme 6: Importance of a clear knowledge of the patient's history

Patients repeatedly cited the key importance of the new physician having in-depth knowledge not just of their current issues but also of their past history.

Topic 4: Factors felt to help transition

7: Friendliness/knowledge

Key factors aiding the transition again included the sense of friendliness and empathy on the first meeting with the new physician as well as a clear demonstration the new physician was aware of the patient's medical history and active issues.

Theme 8: Prior opportunity to meet the new physician

Several patients who had had the opportunity to meet the incoming physician prior to the transition noted that this was very important and allayed anxiety both leading up to the transition and on the first visit. Some who had not had this opportunity suggested that it would have been helpful.

\section{Topic 5: Overall impact on care}

Theme 9: Minimal impact on care

The resounding message in the interviews was that there was no perceived impact on quality of care or confidence in their medical management, even in those who had initially been worried. Only one patient expressed distress with the transition, for reasons largely centered on differing styles of practice, with the rest noting a lack of impact and a few even feeling happier with their care post-transition.

Topic 6: Suggestions for future transitions

The vast majority of patients expressed satisfaction with 
the transition process. When asked for suggestions for future transitions, patients highlighted the importance of understanding why a change was occurring and ideally knowing this up front and in person. Other important aspects of the transition that were mentioned included meeting the incoming physician prior to their first visit and the new physician being well prepared for the first visit. No suggestions beyond those implemented as part of the transition were noted but several of the procedures were reinforced as having importance.

\section{Discussion}

Very little is known about patients' perceptions of COC in the palliative care context. Some studies have examined the concept in the setting of family medicine, geriatrics, and advanced cancer $[17,21,22]$, but have not specifically addressed the impact that changes in physician providers have on patients' perceptions of their care. The focus of this study was to explore patients experiences of a scheduled change in their palliative care physician within the clinic setting, and to assess whether the steps taken to try to mitigate the effect of the change were in fact of benefit.

Our study addressed 6 topics related to the transition experience, each generating its own themes: 1) How change was communicated, 2) Reaction on hearing about the change, 3) Feelings after meeting the new physician, 4) Factors felt to help the transition, 5) Overall impact on care, 6) Suggestions for future transitions.

Although clinic staff, including physicians, were initially concerned that patients would perceive deficiencies in COC, the results of our study indicate that the transition experience was generally perceived as a well-managed and positive process.

Patients expressed satisfaction that they were informed of the upcoming physician in advance of the change and were reassured by in-person communication from the current provider.

"I was well prepared by Dr. A that this would happen. And I was given reasons why this would happen. So knowing that, 1 wasn't bothered"

One patient expressed great appreciation for being introduced to the new physician in advance of the change.

"I thought it was so nice that Dr. A brought Dr. B to see me in clinic. Because it just made the transition very nice".

One patient expressed anxiety over being made aware of the upcoming transition too far in advance and felt that it added to her worry, which leaves open the question of the ideal timing for communication of impending changes. Several patients expressed that knowing 'up front' (ie their initial clinic visit) would have been most helpful whereas many felt that the timing used (4-8 weeks before the change) was just right.

Several patients noted feeling apprehensive about the upcoming change, but subsequently experienced the transition as a positive process.

"The anticipation of a new doctor was more negative than the reality of the change" An understanding of why the change was occurring was also seen as important and reassuring.

"She didn't anticipate any problems with the transition and she was very upfront about it and I had no reason to be concerned or alarmed. It was just very natural and I understood the reason behind it."

Many patients commented on the importance of the behaviours of the incoming physicians. Expressions of attentiveness, friendliness and an empathetic demeanor were appreciated and noted to facilitate the transition.

"She was lovely and smiling. Just her friendliness and the way she introduced herself and having the files and knowing about me and just immediately taking me from where I left off with Dr. A. It just rolled over that it was not a different person"

Empathy and compassion exhibited by the new physician helped the transition:

"Number one is his demeanor, you know very friendly. When he came in he shook my hand and he smiled and made eye contact. Uh, that's all very important. Secondly, he asked if I knew why he was there rather than Dr. A. So he was just confirming my understanding of the change to make sure I was aware of the change and wasn't alarmed that he was there."

Overall the impact of the transition on COC appeared to be at worst neutral though for some it was actually positive.

"I have found the differences had absolutely no impact on the care that I receive from them. They're both excellent"

'sometimes it's a good thing because they bring up things that the other one didn't think about.'

"I didn't want the change, but when it came, I, uh, it came as a pleasant surprise and a welcoming one, after meeting Dr B."

Haggerty et al [5] established a conceptual framework related to COC, which is comprised of three components: relational, informational, and management continuity. Relational continuity refers to an ongoing therapeutic relationship between patients and providers. Informational continuity focuses on the importance of information, either disease or person focused, that links care between providers. Management continuity often assumes particular importance in patients living with chronic or complex illnesses, which requires coordinated and timely delivery of services from multiple providers. Of these, relational continuity has been found to be a particular priority for certain patient populations, including those with chronic health conditions and those receiving home palliative care services [23].

Dimensions of relational continuity include consistency of personnel and an ongoing patient-provider relationship. In their meta-analysis of patients' perceptions of COC, Waibel et al [23] further define elements that facilitate relational continuity, such as being attended by the same physician, continuing with a relationship over time, and quality of the medical consultation provided, including attentiveness, confidence, and medical 
knowledge. Consistency of personnel was cited as an important factor in developing authentic relationships that are required to inspire mutual trust and understanding.

The results of our study indicate that relational continuity can be maintained in physician transitions when they are managed with specific attention to particular elements. Firstly, a focus on establishing rapport and empathy from the initial visit with the new physician may have counteracted the disruption in relational continuity. In addition, those who met the incoming physician during their final clinic visit prior to the transition uniformly highlighted the comfort and smooth transition that the interaction provided. Therefore, a personal introduction seemed a key element in mitigating anxiety as well as ensuring a smooth handover prior to the change in physician provider.

In addition, maintaining informational continuity between providers seemed an important element to foster the patient's trust in the incoming physician.

"It's as if I picked up and left off, because she had obviously read the notes, and I believe they might have even talked personally."

Reid et al [24] describe "information transfer", which refers to the patient's perception of information transfer between providers, as a discrete dimension of informational continuity. Patients in our study clearly valued that incoming physicians had reviewed their medical history prior to the visit and could demonstrate a good understanding of relevant issues even at the initial encounter.

In their study exploring provider's perception of COC, Price et al [25] introduced the concept of "provider connectedness", which describes the cohesiveness of relationships between providers, as an additional factor in an expanded model of COC. Healthcare providers themselves clearly indicated that having established working relationships with colleagues, as well as working in close physical proximity to other providers, are both elements that foster provider connectedness. The structure of our palliative care team allows for a high level of provider connectedness in that team members work out of a common space and generally have daily contact with one another. Specific attention was still paid to direct handover of patient information between providers prior to the transition. Our results support the importance of direct and timely communication between physicians as part of the transition process.

In all, despite concerns within the team that a change in palliative care provider would be troublesome for patients, the overall perception by patients was of minimal impact on the quality and continuity of care, and in several cases, the change was viewed positively. Key factors identified by the patients that eased the transition included the following:

Advanceknowledge of the transition, including an explanation of why it was occurring and an opportunity to discuss it in person with their 'usual' provider.

2. The opportunity to meet the incoming physician at a final visit prior to the transition.

3. A friendly, interested and empathic demeanor by the new physician on the first visit.

4. A clear demonstration of comprehensive handover of information between providers, with the 'new physician' having a strong grasp on key historical and current patient issues.

Limitations of this study include that it was a single site only and included only English speaking patients. In addition pretransition anxiety was likely underestimated by this study given the interview occurred after meeting the new physician. Finally, despite reassurances by the research assistant that responses were confidential, patients may have been reluctant to disclose negative comments while still being followed actively by the clinic staff.

\section{Conclusions}

Our results indicate that the factors contributing to a successful transition in physician care include patients being notified in advance and in-person by the current provider. The physician assuming care should focus on demonstrating a friendly demeanor and thorough knowledge of the patient history at the first encounter. The majority of patients maintained trust in their physicians after the transition and experienced the change positively. These finding suggest that with a focus on certain aspects of relational and informational continuity, patient's perceptions were that overall COC did not suffer.

\section{References}

1. Luckett T, Phillips J, Agar M, Virdun C, Green A, Davidson PM Elements of effective palliative care models: a rapid review. BMC Health Services Research 2014;14:136-158, doi:10.1186/1472-696314-136.

2. Hughes MT, Smith TJ. The growth of palliative care in the United States. Annual Review of Public Health 2014;35:459-475, doi: 10.1146/ annurev-publhealth-032013-182406.

3. Meier D and Beresford L.Outpatient clinics are a new frontier for palliative care. J Pall Med 2008;11(6):823-828, doi: 10.1089/ jpm.2008.9886.

4. Rabow M, Kvale E, Barbour L, Cassel JB, Cohen S, Jackson V, et al. Moving upstream: a review of the evidence of the impact of outpatient palliative care. J Pall Med 2013;16(12):1540-1549, doi: 10.1089/ jpm.2013.0153.

5. Haggerty JL, Reid RJ, Freeman GK, Starfield BH, Adair CE, McKendry R. Continuity of care: a multidisciplinary review. BMJ 2003;327(7425):1219-1221.

6. Dy SM, Apostol C, Martinez KA, Aslakson RA.Continuity, coordination and transitions of care for patients with serious and advanced illness: a systematic review of interventions. J Pall Med 2013;16(4):436445, doi: 10.1089/jpm.2012.0317.

7. Roland M. Continuity of care: betrayed values or misplaced nostalgia. International Journal of Integrated Care 2012;12: 1-4.

8. Borgsteede SD, Graafland-Riedstra C, Deliens L, Francke AL, van Eijk JT, Willems DL. Good end-of-life care according to patients and their GPs. British Journal of General Practice 2006;56(522):20-26. 
9. Heyland DK, Dodek, P, Rocker G, Groll D, Gafni A, Pichora D, et al. What matters most in end-of-life care: Perceptions of seriously ill patients and their family members. CMAJ 2006;174(5):627-633.

10. Leydon GM, Shergill NK, Campion-Smith C, Austin H, Eyles C Baverstock J, et al. Discontinuity of care at end of life: a qualitative exploration of $\mathrm{OOH}$ end of life care. BMJ Support Palliat Care 2013;3:412-21, doi:10.1136/bmjspcare-2012-000266.

11. Michiels E, Deschepper R, Van Der Kelen G, Bernheim JL, Mortier F, Vander Stichele R, Deliens L. The role of general practitioners in continuity of care at the end of life: a qualitative study of terminally ill patients and their next of kin. Palliat Med. 2007 Jul;21(5):409-415.

12. Aubin M, Giguere A, Martin M, Verreault R, Fitch MI, Kazanjian A et al. Interventions to improve continuity of care in the follow-up of patients with cancer (review). Cochrane Database of Sytematic Reviews 2012;7, doi: 10.1002/14651858.CD007672.

13. O'Mahony A, Blank AE, Zallman L, Selwyn PA. The benefits of a hospitalbased npatient palliative care consultation service: preliminary outcome data. Journal of Palliative Medicine 2005;8(5):1033-1039.

14. Hanks GW, Robbins M, Sharp D, Forbes K, Done K, Peters TJ, et al. The impact study: a randomized controlled trial to evaluate a hospital palliative care team. Br. J. Cancer 2002;87(7):733-739.

15. Temel JS, Greer JA, Muzikansky A, Gallagher ER, Admane S, Jackson VA, et al. Early palliative care for patients with metastatic non-smallcell lung cancer. N. Engl. J. Med 2010;363(8):733-742, doi: 10.1056/ NEJMoa1000678.

16. Zimmerman C, Swami N, Rodin G, Hannon B, Rydall A, Donner A, et al. Early palliative care for patients with metastatic cancer : a clusterrandomized controlled trial. The Lancet 2014; 383:1721-1730.

17. Hussain A, Barbera L, Howell D, Moineddin R, Bezjak A, Sussman J. Advanced lung cancer patients' experience with continuity of care and supportive care needs. Support Care Cancer 2013;21(5):13511358, doi: 10.1007/s00520-012-1673-7.
18. Schers H, Webster S, van den Hoogen H, Avery A, Grol R, van den Bosch W. Continuity of care in general practice: a survey of patients views. Br J Gen Pract 2002;52(479):459-462.

19. Stokes T, Tarrant C, Mainous A, Schers H, Freeman G, Baker R,et al. Continuity of care: is the personal doctor still important? A survey of general practitioners and family physicians in England and Wales, the United States, and the Netherlands. Ann Fam Med 2005;3(4):353-359.

20. Randall E, Crooks VA, Goldsmith LJ. In search of attachment: a qualitative study of chronically ill women transitioning between family physicians in rural Ontario, Canada. BMC Fam Pract 2012;13:125137, doi:10.1186/1471-2296-13-125.

21. Soelver L, Rydahl-Hansen S, Oestergaard B, Wagner L. Identifying factors significant to continuity in basic palliative hospital care-from the perspective of patients with advanced cancer. Journal of Psychosocial Oncology 2014;32(2):167-188,doi: 10.1080/07347332.2013.873999.

22. King M, Jones L, Richardson A, Murad S, Irving A, Aslett H, et al. The relationship between patients' experiences of continuity of cancer care and health outcomes: a mixed methods study. British Journal of Cancer 2008;98(3):529-536, doi: 10.1038/sj.bjc.6604164.

23. Waibel S, Henao D, Aller MB, Vargas I, Vázquez ML. What do we know about perceptions of continuity of care? Int J Qual Helath Care 2012;24:39-48

24. Reid R, Haggerty J, McKendry R. Defusing the confusion: concepts and measures continuity in healthcare. Ottawa: Canadian Health Services Foundation;2002

25. Price M, Lau FY. Provider connectedness and communication patterns: extending continuity of care in the context of circle of care. BMC Health Services Research 2013; 13:309-409, doi: 10.1186/14726963-13-309. 